\title{
Avaliação econômica de tecnologia nos serviços de saúde do Brasil: uma revisão de
}

\author{
escopo \\ Health evaluation of technologies in health services in Brazil: a scoping review \\ Evaluación económica de tecnología en servicios de la salud brasileña: una revisión de alcance
}

Recebido: 27/11/2021 | Revisado: 01/12/2021 | Aceito: 10/12/2021 | Publicado: 18/12/2021

Francisco Luciano Cavalcanti Filho

ORCID: https://orcid.org/0000-0003-0665-9626 Universidade Estadual do Ceará, Brasil

E-mail: lucianocavalcanti16@hotmail.com

Maria Salete Bessa Jorge

ORCID: https://orcid.org/0000-0001-6461-3015 Universidade Estadual do Ceará, Brasil E-mail: maria.jorge@uece.br

Cybelle Façanha Barreto Medeiros Linard

ORCID: https://orcid.org/0000-0001-7927-9320 Universidade Estadual do Ceará, Brasil E-mail: cybellelinard@yahoo.com.br

\begin{abstract}
Resumo
Objetivo: Mapear as principais avaliações econômicas de tecnologias utilizadas nos serviços de saúde do Brasil. Método: Trata-se de uma revisão scoping review, com pesquisa nos bancos de dados, com inclusão dos artigos publicados até o dia 18 de julho de 2021. Foram incluídos estudos com AES, com indicadores econômicos, de resultados e custos. Foram excluídos estudos com ausência de dados econômicos, sem análises econômicas comparativas, revisões de literatura, artigos com textos indisponíveis, pareceres e decisões de órgãos governamentais, estudos desenvolvidos fora do Brasil. Resultados: Ao final da seleção 10 estudos foram considerados na presente revisão. Discussão: Os artigos apresentam dados relevantes, contudo faz-se necessário evoluir com a utilização dos conceitos da economia da saúde para uma metodologia eficaz quanto aos objetivos das AES. As AES oferecem aos gestores efetivas evidências para decisões, otimizando a aplicação dos recursos, oferecendo uma assistência com maior segurança e qualidade, e melhorando o acesso ao sistema de saúde. Considerações finais: É fundamental incluir nas próximas AE valores como curas, retorno antecipado a atividade econômica, tempo de internação; e valoração de $\mathrm{RH}$, custos com performance dos dispositivos avaliados.
\end{abstract}

Palavras-chave: Avaliações econômicas; Tecnologia; Serviços de saúde do Brasil; Organizações de saúde.

\begin{abstract}
Goal: To outline the main health evaluation (HE) technologies used in health services in Brazil. Method: scoping review in databases, with papers published until July 18 2021. Papers with health evaluation and costs, results and economic indexes were included. All the papers with none of the aforementioned components and literature reviews, unavailable texts, legal opinions and decisions from governmental agencies and papers developed outside Brazil were excluded. Results: In the end, 10 papers were considered for this review. Discussion: The papers showed relevant data, although an improvement of the utilization of health economy concepts are needed for a better methodology in health evaluation. Health evaluation provide efficient evidences for decisions, improving resource deployment, offering safer and better health services, also enhancing health services accessibility. Conclusions: It is vital to include values such as HR appraisal, early return to work, cure, length of stay and costs of evaluated devices in the next health evaluations.
\end{abstract}

Keywords: Health evaluation; Technology; Health services in Brazil; Health organizations.

\section{Resumen}

Objetivo: Mapear las principales evaluaciones económicas de tecnologías utilizadas en los servicios de salud en Brasil. Método: Se trata de una revisión de alcance, con búsqueda en bases de datos, incluyendo artículos publicados hasta el 18 de julio de 2021. Se incluyeron estudios con evaluaciones económicas, con indicadores económicos, resultados y costos. Se excluyeron los estudios sin datos económicos, sin análisis económicos comparativos, revisiones de literatura, artículos con textos no disponibles, opiniones y decisiones de agencias gubernamentales, estudios desarrollados fuera de Brasil. Resultados: Al final de la selección, se consideraron 10 estudios en esta revisión. Discusión: Los artículos presentan datos relevantes, sin embargo es necesario evolucionar con el uso de conceptos de economía de la salud para una metodología efectiva en cuanto a los objetivos de la AES. Las AES 
ofrecen a los gestores evidencia eficaz para la toma de decisiones, optimizando la aplicación de los recursos, ofreciendo una atención con mayor seguridad y calidad y mejorando el acceso al sistema de salud. Consideraciones finales: Es fundamental incluir en la próxima EA valores como curas, retorno temprano a la actividad económica, duración de la estadía; y valoración de RRHH, costes de rendimiento de los dispositivos evaluados.

Palabras clave: Valoraciones económicas; Tecnología; Servicios de salud en Brasil; Organizaciones sanitarias.

\section{Introdução}

A relação entre saúde e economia pode ser estudada com várias perspectivas, uma delas é que não existe crescimento econômico sem capital humano, uma outra é que os gestores do setor de saúde necessitam de evidências representadas por indicadores de processos e de resultados em saúde para as tomadas de decisões; em um contexto de muitas necessidades e recursos escassos, a economia da saúde oferece como ferramenta a avaliação econômica, que busca oferecer através de evidências opções alternativas diversas para tomada de decisão, responder perguntas de como melhor empregar os recursos do sistema de saúde torna-se uma tarefa difícil de ser executada sem avaliações de alternativas possíveis.

Os prestadores de serviços hospitalares do sistema de saúde possuem o grande desafio de cuidar dos pacientes que buscam seus serviços, promover a cura desses pacientes e devolvê-los para a sociedade com sua capacidade física e mental plena, com capacidade economicamente ativa, são seus principais objetivos. A Diretoria Colegiada da Anvisa publicou a resolução 36, de 25 de julho de 2013, que instituiu ações aplicáveis aos serviços de saúde , públicos e privados, com objetivo de promover a segurança do paciente e a melhoria da qualidade da assistência; essa mesma resolução determina que as diretorias dos serviços de saúde criem os núcleos de segurança do paciente que passam a ter a responsabilidade e autoridade para executar as ações do plano de segurança do paciente em serviços de saúde; o plano tem por objetivo estabelecer estratégias e ações de gestão de risco, entre elas estão: a segurança na prescrição, uso e administração de medicamentos; segurança no uso de equipamentos e materiais; prevenção e controle de eventos adversos em serviços de saúde, incluindo as infecções relacionadas à assistência à saúde. Esse tema é tão importante no contexto do cuidado com o paciente que a mesma resolução enquadra o descumprimento da resolução como infração sanitária, sem prejuízo das responsabilidades civil, administrativa e penal cabíveis ${ }^{1}$.

Nesse contexto os gestores das organizações de saúde necessitam de conhecimento científico, inteligência e empoderamento para decidirem sobre quais estratégias, táticas, ações, recursos e intervenções precisam escolher para emprego dos recursos disponíveis e alcançar seus objetivos, e para essa jornada se faz necessário observar eventos chaves do conhecimento como a valoração dos recursos que necessitam serem efetivas em custos.

Com o propósito de contribuir com uma melhor gestão dos recursos do sistema de saúde, utilizando as inteligências da economia da saúde, este estudo tem como objetivo mapear as principais avaliações econômicas de tecnologia utilizadas nos serviços de saúde do Brasil.

\section{Metodologia}

Utilizou-se do método scoping review, que tem como base os cinco estágios estabelecidos por Arksey \& O’Malley ${ }^{2}$ : 1) identificação da questão de pesquisa; 2) identificação de estudos relevantes; 3) seleção dos estudos; 4) mapeamento dos dados e 5) agrupamento, resumo e relato dos resultados. Para elaboração da revisão, seguiram-se as recomendações do checklist PRISMA-ScR (PRISMA extension for Scoping Review) ${ }^{3}$.

Com o uso do acrônimo PCC, foi formulada a questão da pesquisa, em que o P (população) é representado pelo termo avaliações econômicas; o C (conceito) pelo termo tecnologia; e o C (contexto) pelo termo serviços de saúde do Brasil ${ }^{4}$. Com isso, estabeleceu-se a seguinte pergunta problema: quais as principais avaliações econômicas de tecnologia em saúde são utilizadas nos serviços de saúde do Brasil? 
Após pesquisas nos bancos de dados DECS, MeSH e Emtry, foram definidos os seguintes descritores: avaliação em saúde, avaliação econômica em saúde, impacto no estado de saúde, health evaluation, evaluación en salud, benchmarking, cost-benefit analysis, benefits and costs, tecnologia, technology, technol, administração de serviços de saúde, health services administration, administración de los servicios de salud, organizações de assistência responsáveis, accountable, Care organizations, organizaciones responsables por la atención, marketing de serviços de saúde, marketing of health services, comercialización de los servicios de salud, economia e organizações de saúde, health care economics and organizations, economía y organizaciones para la atención de la salud, serviços de saúde, health services, servicios de salud, hospitais, hospitals, hospitales, health service, services, health.

Uma estratégia de busca detalhada, e individual, foi aplicada em cada banco de dados: PubMed, Web of Science, Scopus e Biblioteca Virtual de Saúde (BVS). Sendo incluídos todos os artigos publicados até o dia 18 de julho de 2021 , sem restrição de tempo.

Durante a estratégia de busca, foram utilizados como critérios de inclusão todos os tipos de estudos com avaliações econômicas, independente das características e doenças da população; estudos que tenham métodos de avaliação econômica, que apresentem indicadores econômicos, e que tenham análises de custo efetividade, de custo utilidade, de custo-benefício, de custo minimização, de custo oportunidade, ou quaisquer outras análises econômicas com indicadores de resultados e custos. Foram excluídos estudos com ausência de dados econômicos; estudos com apenas preços das tecnologias, sem metodologia e análises econômicas comparativas, sem evidências com resultados valorados; cartas com opiniões pessoais sem evidências econômicas; protocolos; editorias; revisões sistemáticas e quaisquer outros tipos de revisões de literatura; artigos cujos conteúdos na íntegra não estejam disponíveis; pareceres e decisões técnicas emitidos por órgãos governamentais; estudos desenvolvidos fora do Brasil; estudos escritos em linguagem que não usem o alfabeto latino romano.

A apropriada combinação dos termos foi selecionada e adaptada para cada base de dados (Quadro 1). O software Mendeley (Mendeley Ltda) foi utilizado para o manejo de todas as referências e a remoção dos artigos duplicados. A seleção dos estudos foi realizada em quatro etapas, por dois revisores independentes.

Quadro 1 - Equações de buscas nos bancos de dados.

\begin{tabular}{|c|c|}
\hline BASE & ESTRATÉGIA DE BUSCAS \\
\hline $\begin{array}{l}\text { PubMed, } \\
\text { Scopus, } \\
\text { BVS }\end{array}$ & $\begin{array}{l}\text { ("avaliação em saúde" or "health evaluation" or "evaluación en salud" or "avaliação econômica em saúde" or "impacto no estado de saúde" or } \\
\text { benchmarking or "cost-benefit analysis" or "benefits and costs" or "avaliação comparativa" or "cost benefit" or "cost benefit analysis" or "cost } \\
\text { effectiveness" or "cost-benefit data" or "cost-effectiveness analysis" or "cost-utility analysis" or "costs and benefits" or "economic evaluation" } \\
\text { or "marginal analysis previous indexing" or "costs and cost analysis") AND ("tecnologia or technology or technol") AND ("administração de } \\
\text { serviços de saúde" or "health services administration" or "administración de los servicios de salud" or "organizações de assistência } \\
\text { responsáveis" or "accountable care organizations" or "organizaciones responsables por la atención" or "marketing de serviços de saúde" or } \\
\text { "marketing of health services" or "comercialización de los servicios de salud" or "economia e organizações de saúde" or "health care } \\
\text { economics and organizations" or "economía y organizaciones para la atención de la salud" or "serviços de saúde" or "health services" or } \\
\text { "servicios de salud" or hospitais or hospitals or hospitales or "health service" or "services, health" or "organizações responsáveis pela } \\
\text { assistência" or "organizações responsáveis de prestadores de serviços de saúde" or "mercado de serviços de saúde" or "economia da } \\
\text { assistência à saúde" or "economia da atenção à saúde" or "economia da saúde" or "economia dos cuidados de saúde" or "rede prestadora de } \\
\text { serviços de saúde" or "serviço de saúde") AND ("brasil or brazil") }\end{array}$ \\
\hline $\begin{array}{l}\text { Web of } \\
\text { Science }\end{array}$ & $\begin{array}{l}\text { \#1 : TS = ("avaliação em saúde" or "health evaluation" or "evaluación en salud" or "avaliação econômica em saúde" or "impacto no estado de } \\
\text { saúde" or benchmarking or "cost-benefit analysis" or "benefits and costs" or "avaliação comparativa" or "cost benefit" or "cost benefit } \\
\text { analysis" or "cost effectiveness" or "cost-benefit data" or "cost-effectiveness analysis" or "cost-utility analysis" or "costs and benefits" or } \\
\text { "economic evaluation" or "marginal analysis previous Indexing" or "costs and cost analysis") } \\
\text { \#2:TS = ("tecnologia or technology or technol ") } \\
\text { \#3: TS = ("administração de serviços de saúde" or "health services administration" or "administración de los servicios de salud" or } \\
\text { "organizações de assistência responsáveis" or "accountable care organizations" or "organizaciones responsables por la atención" or } \\
\text { "marketing de serviços de saúde" or "marketing of health services" or "comercialización de los servicios de salud" or "economia e } \\
\text { organizações de saúde" or "health care economics and organizations" or "economía y organizaciones para la atención de la salud" or "serviços } \\
\text { de saúde" or "health services" or "servicios de salud" or hospitais or hospitals or hospitales or "health service" or "services, health" or } \\
\text { "organizações responsáveis pela assistência" or "organizaçóes responsáveis de prestadores de serviços de saúde" or "mercado de serviços de } \\
\text { saúde" or "economia da assistência à saúde" or "economia da atenção à saúde" or "economia da saúde" or "economia dos cuidados de saúde" } \\
\text { or "rede prestadora de serviços de saúde" or "serviço de saúde") } \\
\text { \#4 : TS = ("brasil or brazil ") } \\
\text { \#1 AND \#2 AND \#3 AND \#4 }\end{array}$ \\
\hline
\end{tabular}


Na primeira etapa foi aplicado a equação de busca e capturados todos os estudos que atenderam os critérios da equação.

Na segunda etapa foram excluídos os estudos duplicados, atividade realizada através do gerenciador Mendeley.

$\mathrm{Na}$ terceira etapa foi realizada uma análise do potencial do estudo pela leitura do título, resumo e resultados.

Na quarta etapa foi aplicado os critérios de inclusão e exclusão no conteúdo, e realizado a elegibilidade dos estudos para a análise.

Foi construído um formulário para a extração e o gerenciamento das informações, sendo realizado de acordo com os aspectos de um roteiro de observação e mapeamento. As informações foram organizadas por meio de um quadro sinóptico (Quadro 2) com a descrição dos seguintes aspectos: autor, ano, local do estudo, tipo de avaliação econômica, perspectiva do estudo quanto a organização de saúde alcançada, organização de saúde responsável, tecnologia intervenção, tecnologia controle, população, tipo e/ou método do estudo, horizonte de tempo, resultados econômicos de dominância.

Procedeu-se, a seguir, à leitura dos artigos, visando organizar os dados em categorias temáticas, conforme a semelhança e mediante a abordagem e estatística descritiva. Os resultados obtidos foram discutidos e interpretados com base em autores que publicaram sobre o tema.

Este artigo é parte de dissertação de Mestrado em Gestão de Saúde (MEPGES), como parte dos requisitos para se obter o título de mestre em gestão em saúde pela UECE (Unversidade Estadual do Ceará)

\section{Resultados}

\section{Seleção dos estudos}

Um total de 395 estudos foram inicialmente encontrados nos quatro bancos de dados, PubMed, Web of Science, Scopus, BVS. Após a remoção dos artigos duplicados, que somaram um total de 11 estudos, os títulos e os resumos dos artigos foram avaliados, e 50 foram considerados potencialmente relevantes para leitura e avaliação. Ao final da seleção 10 estudos foram considerados na presente revisão. A Figura 01 mostra o fluxograma de identificação, escaneamento, elegibilidade e inclusão adaptado do Preferred Reporting Items for Systematic Review and Meta-Analyses (PRISMA). 
Figura 1 - Fluxograma da seleção das publicações baseado no modelo PRISMA, Fortaleza (CE), Brasil, 2021
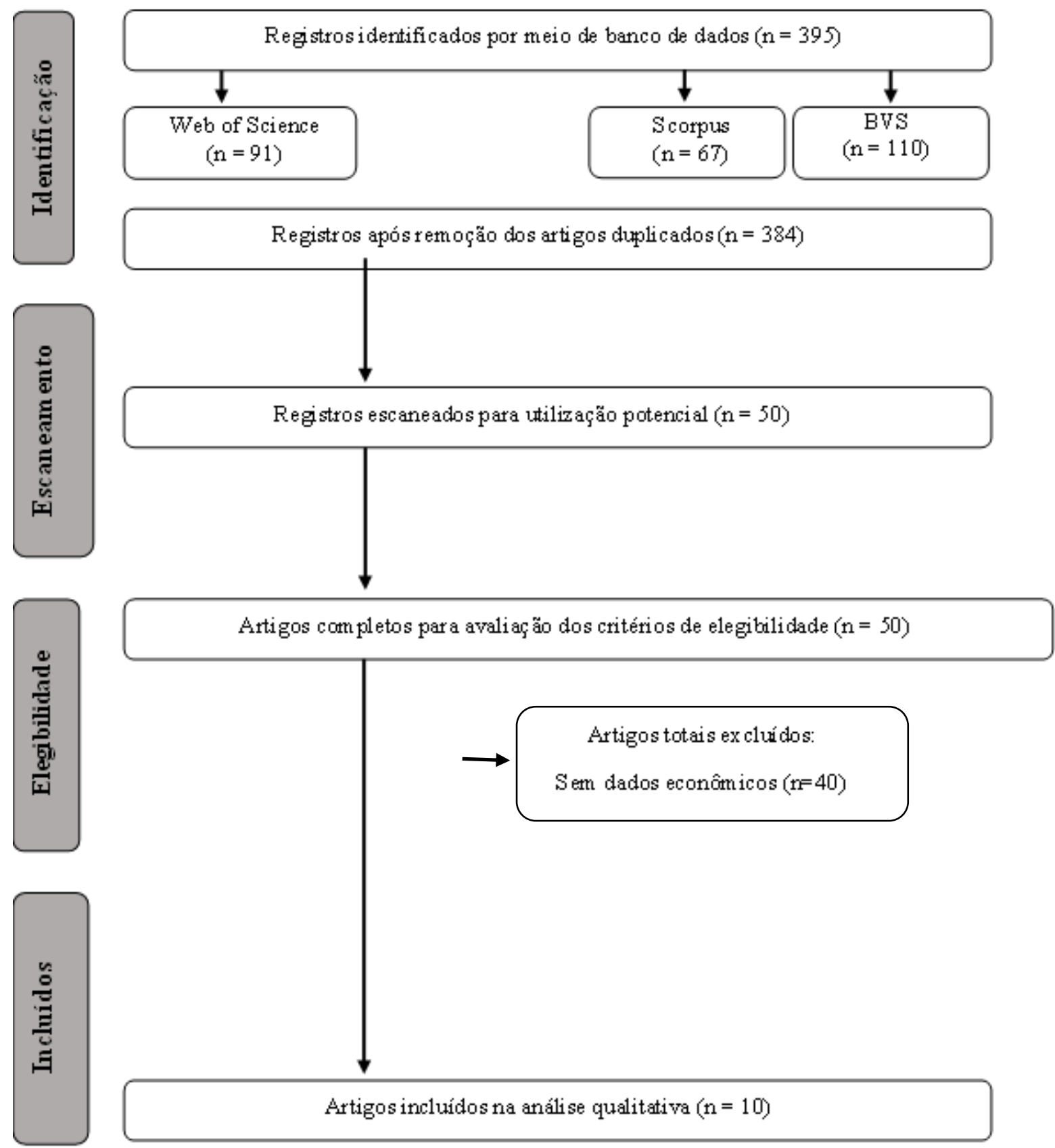

Fonte: Autores.

\section{Perfil dos estudos selecionados}

Foi construído um banco de dados a partir dos estudos selecionados para identificação das semelhanças, temas e lacunas da literatura ${ }^{5}$; análise realizada a partir da extração das principais informações, de um gerenciamento de mapeamento seguindo um roteiro de observação (Quadros 2 e 3). 
Research, Society and Development, v. 10, n. 16, e536101623990, 2021

(CC BY 4.0) | ISSN 2525-3409 | DOI: http://dx.doi.org/10.33448/rsd-v10i16.23990

\begin{tabular}{|c|c|c|c|c|c|c|c|}
\hline $\begin{array}{c}\mathrm{Nr} \\
\text { Controle }\end{array}$ & Autor & Ano & $\begin{array}{c}\text { Local de } \\
\text { Desenvolvimento }\end{array}$ & $\begin{array}{c}\text { Tipo de } \\
\text { Avaliação } \\
\text { Econômica }\end{array}$ & $\begin{array}{l}\text { Perspectiva do } \\
\text { Estudo }\end{array}$ & $\begin{array}{l}\text { Organização de Saúde } \\
\text { Base do Estudo }\end{array}$ & Tipo de Intervenção \\
\hline 1 & $\begin{array}{l}\text { Veras, Bruna Medeiros; Senna, Katia Marie Correia; Marcelo } \\
\text { Goulart; Santos, Marisa Silva }^{6}\end{array}$ & 2013 & RJ & ACE & $\begin{array}{l}\text { Hosp Público } \\
\text { Federal }\end{array}$ & $\begin{array}{c}\text { Instituto Nacional de } \\
\text { Cardiologia / RJ }\end{array}$ & Cateteres Cardíacos Reusáveis \\
\hline 2 & $\begin{array}{l}\text { Meirelles, Isandra Oliveira; Couto, Dulce Helena Nunes; Costa, } \\
\text { Rodrigo Saar da }\end{array}$ & 2019 & RJ & ACE & $\begin{array}{c}\text { Hosp Público } \\
\text { Federal } \\
\end{array}$ & INCA / RJ & Pazopanibe \\
\hline 3 & $\begin{array}{l}\text { Ubels, Jasper; Sable, Craig; Beaton, Andrea Z Nunes, Maria Carmo } \\
\text { P; Oliveira, Kaciane K B Rabelo, Lara C; Teixeira, Isabella M; Ruiz, } \\
\text { Gabriela Z L; Rabelo, Letícia Maria M; Tompsett, Alison R; Ribeiro, } \\
\text { Antonio Luiz P; Sahlen, Klas-Göran; Nascimento, Bruno R }{ }^{8}\end{array}$ & 2017 & MG & ACE & População MG & HC UFMG & $\begin{array}{l}\text { Triagem ecocardiográfica da Doença } \\
\text { Cardíaca Reumática (RHD) utilizando } \\
\text { dispositivos portáteis }\end{array}$ \\
\hline 4 & $\begin{array}{l}\text { Mattos, Ângelo Zambam de Mattos, Angelo Alves de Ribeiro, } \\
\text { Rodrigo Antonini }{ }^{9}\end{array}$ & 2016 & RS & ACM & SUS & $\begin{array}{c}\text { Programa de Pós Graduação } \\
\text { da UFRS }\end{array}$ & Terlipressin \\
\hline 5 & Oliveira, Renato Almeida Rosa de ${ }^{10}$ & 2019 & SP & $\begin{array}{l}\text { ACE, ACU, } \\
\text { ACM }\end{array}$ & AC Camargo & $\begin{array}{l}\text { Pós Graduação Doutorado - } \\
\text { Fund Antônio Prudente }\end{array}$ & $\begin{array}{l}\text { Programa de rastreamento da população } \\
\text { com câncer de próstata ; implementação } \\
\text { de novas tecnologias no tratamento }\end{array}$ \\
\hline 6 & Gomes, Marcelo; Romcy, Helena ${ }^{11}$ & 2018 & SP & ACE & $\begin{array}{l}\text { Operadoras da } \\
\text { Saúde } \\
\text { Suplementar }\end{array}$ & $\begin{array}{l}\text { Sense Company com dados } \\
\text { do HC de SP }\end{array}$ & $\begin{array}{l}\text { Seringa preenchida para flush pronto para } \\
\text { uso }\end{array}$ \\
\hline 7 & $\begin{array}{l}\text { Correia, MITD; Castro, M; Toledo, D D; Farah, D; Sansone, D; } \\
\text { Andrade, T R D; de Araújo, G T B; Fonseca, M C M }{ }^{12}\end{array}$ & 2020 & MG & ACE & SUS & UFMG & Terapia nutricional \\
\hline 8 & $\begin{array}{l}\text { Peixoto, Henry M; Brito, Marcelo A M; Romero, Gustavo A S; } \\
\text { Monteiro, Wuelton M; de Lacerda, Marcus V G; de Oliveira, Maria } \\
\text { R F }^{13}\end{array}$ & 2016 & DF & $\mathrm{ACE}$ & SUS & Universidade de Brasília & Diagnóstico CS-G6PD \\
\hline 9 & $\begin{array}{l}\text { de Miranda, Mirela Costa; Haddad, Luciana Bertocco de Paiva; } \\
\text { Trindade, Evelinda } \\
\text { Cassenote, Alex; Hayashi, Giselle Y; Damiani, Durval; Costa, } \\
\text { Fernanda Cavalieri; Madureira, Guiomar; de Mendonca, Berenice } \\
\text { Bilharinho } \\
\text { Bachega, Tania A S S }{ }^{14}\end{array}$ & 2021 & SP & ACE & SUS & HC USP & $\begin{array}{c}\text { Triagem neonatal para hiperplasia adrenal } \\
\text { congênita }\end{array}$ \\
\hline 10 & $\begin{array}{l}\text { Marcolino, Milena } \quad \text { Soriano; } \text { Polanczyk, Carisi } \text { Anne } \\
\text { Bovendorp, Ana Carolina Caixeta; Marques, Naiara Silveira; Silva, } \\
\text { Lilian Azevedo da; Turquia, Cintia Proveti Barbosa; Ribeiro, } \\
\text { Antônio Luiz }^{15}\end{array}$ & 2016 & MG & ACM & SUS & Hosp Odilon Behrens & $\begin{array}{l}\text { Anticoagulantes orais: Warfarin, } \\
\text { Apixaban Dabigatran, Rivaroxaban }\end{array}$ \\
\hline
\end{tabular}

Fonte: Autores. 
Research, Society and Development, v. 10, n. 16, e536101623990, 2021

(CC BY 4.0) | ISSN 2525-3409 | DOI: http://dx.doi.org/10.33448/rsd-v10i16.23990

Quadro 3 - Quadro sinóptico dos artigos elegíveis, caraterísticas finais.

\begin{tabular}{|c|c|c|c|c|c|}
\hline $\begin{array}{c}\mathrm{Nr} \\
\text { Controle } \\
\end{array}$ & Tipo de Controle & População Avaliada & Tipo de Estudo e Método & Horizonte de Tempo & Resultados \\
\hline 1 & Cateteres Cardíacos Novos & Pacientes com cateteres de cinecoronariografia & $\mathrm{AC}$ aquisição direta & $\begin{array}{l}\text { Horizonte não } \\
\text { identificado }\end{array}$ & $\begin{array}{l}\text { Relação de custo efetividade incremental favorável } \\
\text { ao reuso (intervenção) }\end{array}$ \\
\hline 2 & Sunitinibe & Pacientes com câncer renal metastático & Modelagem & 10 meses & $\begin{array}{l}\text { Efeito dominante para a intervenção; razão de custo } \\
\text { efetividade incremental para a intervenção }\end{array}$ \\
\hline 3 & Processo manual tradicional & $\begin{array}{c}\text { Pacientes com } 11 \text { anos acompanhadas pelo } \\
\text { Programa de Rastreamento da Valvopatia } \\
\text { Reumática }\end{array}$ & Modelo Markov & 30 anos & Dominância de custo efetividade para a intervenção \\
\hline 4 & Noradrenaline & Pacientes com sindrome hepatorenal & $\begin{array}{l}\text { Análise de sensibilidade } \\
\text { probabilística }\end{array}$ & $\begin{array}{l}\text { Horizonte não } \\
\text { identificado }\end{array}$ & Dominância de custo minimização para intervenção \\
\hline 5 & Não rastrear e não intervir & População com câncer de próstata & $\begin{array}{c}\text { Tese de doutorado: análise de } \\
\text { dados epidemiológicos e } \\
\text { econômicos }\end{array}$ & 5 anos & Dominância para as intervenções \\
\hline 6 & $\begin{array}{l}\text { Procedimento para flush com } \\
\text { seringa preenchida manualmente }\end{array}$ & $\begin{array}{l}\text { Pacientes internados com cateteres vasculares } \\
\text { centrais }\end{array}$ & $\begin{array}{l}\text { Avaliação econômica baseada } \\
\text { em modelo de árvore de } \\
\text { decisão }\end{array}$ & 1 ano & Dominância custo efetivo para a intervenção \\
\hline 7 & Sem a intervenção & População hospitalizada & $\begin{array}{c}\text { Avaliação econômica de } \\
\text { análise de custo efetividade }\end{array}$ & 1 ano & Dominância custo efetivo para a intervenção \\
\hline 8 & BX - G6PD & Pacientes masculinos com malária & Árvore decisória & $\begin{array}{l}\text { Horizonte não } \\
\text { identificado }\end{array}$ & Dominância custo efetivo para a intervenção \\
\hline 10 & $\begin{array}{l}\text { Anticoagulantes orais: Warfarin, } \\
\text { Apixaban Dabigatran, Rivaroxaban }\end{array}$ & $\begin{array}{c}\text { Pacientes } \\
\text { com fibrilação atrial para prevenção de } \\
\text { eventos cardioembólicos. }\end{array}$ & $\begin{array}{l}\text { Análise econômica } \\
\text { comparativa }\end{array}$ & $\begin{array}{l}\text { Horizonte não } \\
\text { identificado }\end{array}$ & Warfarin é dominante frente aos outros coagulantes \\
\hline
\end{tabular}

Fonte: Autores. 
Research, Society and Development, v. 10, n. 16, e536101623990, 2021

(CC BY 4.0) | ISSN 2525-3409 | DOI: http://dx.doi.org/10.33448/rsd-v10i16.23990

Verificou-se que, do total de artigos, $10 \%$ (1) foi publicado em 2013, 30\% (3) publicado em 2016, 10\% (1) publicado em 2017, $10 \%$ (1) publicado em 2018, $20 \%$ (2) publicado em 2019, 10\% (1) publicado em 2020 e 10\% (1) publicado em 2021, assim os artigos publicados estão entre o período de 2013 a 2021, com uma tendência linear de queda no número de publicações, conforme apresentado no Gráfico 1.

Gráfico 1 - Quantidade de estudos de acordo com o ano de publicação.

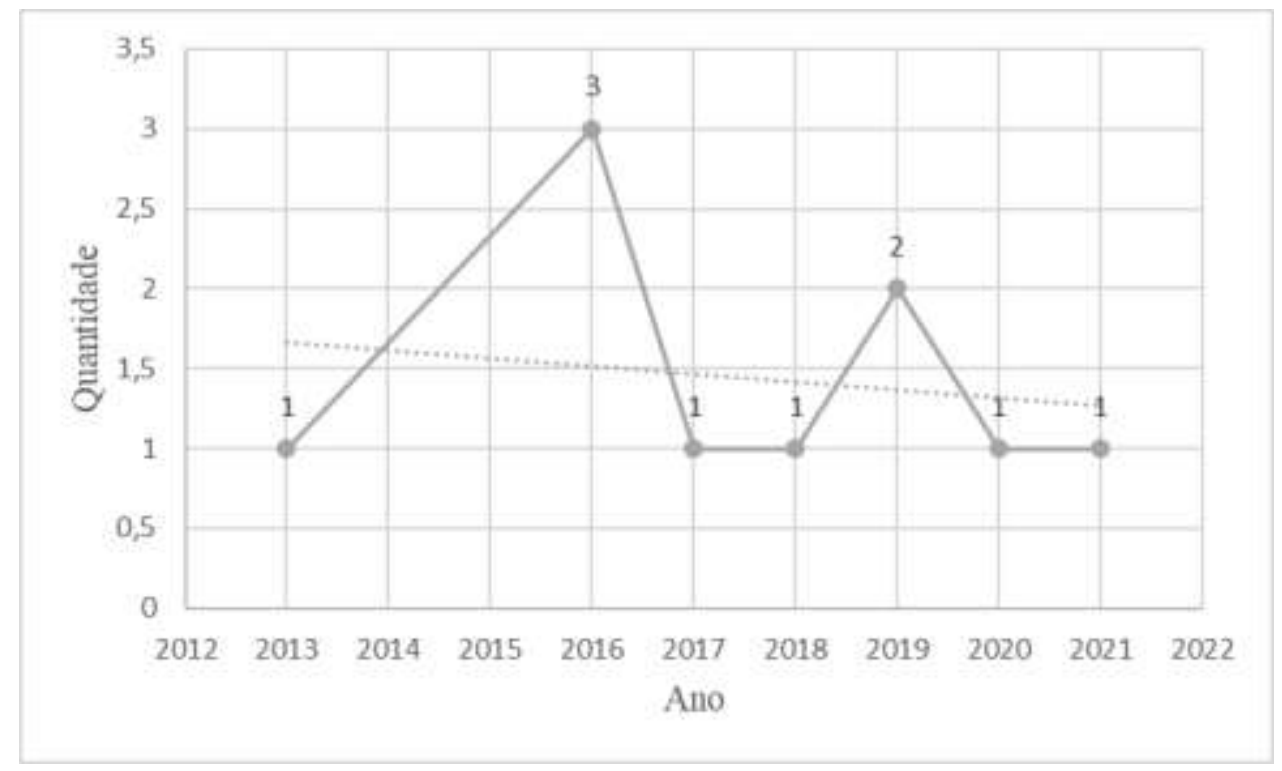

Fonte: Autores.

Pode-se verificar que, do total de artigos publicados, 10\% (1) foi desenvolvido no DF (Distrito Federal), 30\% (3) em MG (Minas Gerais), 20\% (2) no RJ (Rio de Janeiro), 10\% (1) no RS (Rio Grande do Sul), e 30\% (3) em SP (São Paulo), como apresentado no Gráfico 2.

Gráfico 2 - Estados (local) onde foram desenvolvidos os estudos.

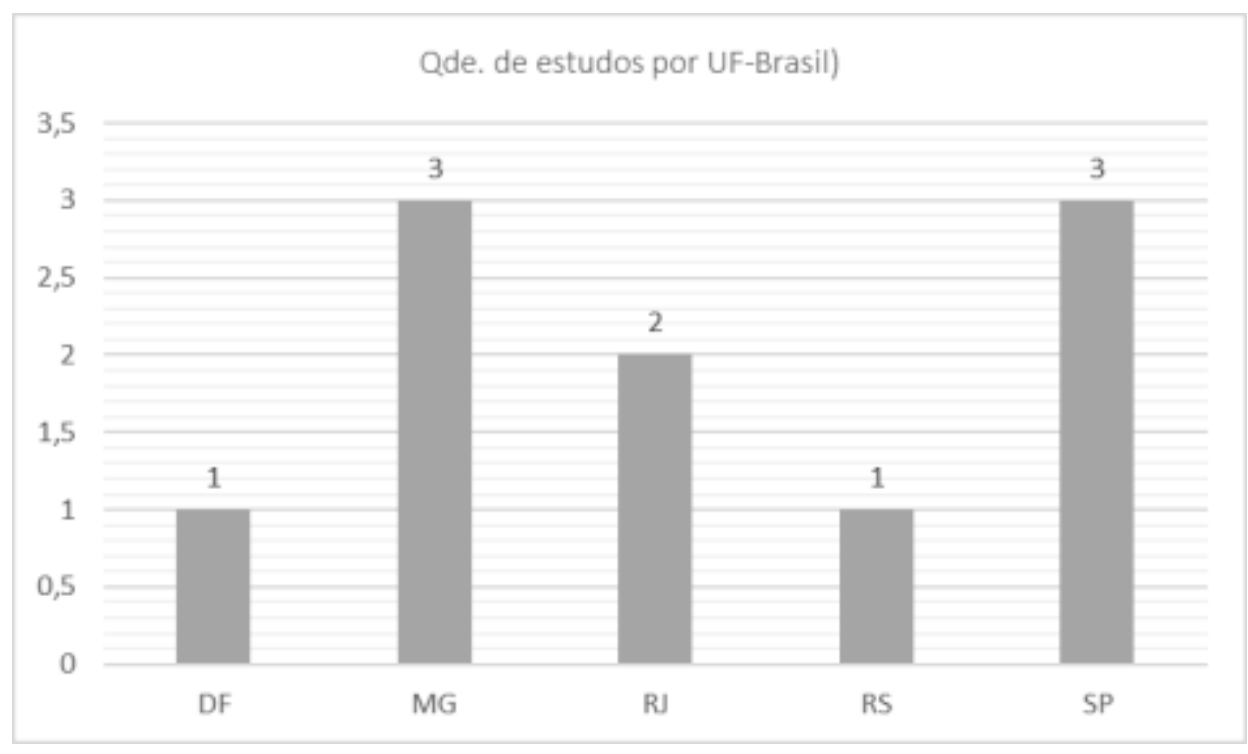

Fonte: Autores. 
Pode-se verificar que, do total de artigos publicados, 10\% (1) foi desenvolvido no DF (Distrito Federal), 30\% (3) em MG (Minas Gerais), 20\% (2) no RJ (Rio de Janeiro), 10\% (1) no RS (Rio Grande do Sul), e 30\% (3) em SP (São Paulo), como apresentado no Gráfico 2.

Observou-se que 80\% (8) dos estudos foram realizados com uma perspectiva do SUS (Sistema Único de Saúde do Brasil), organizações públicas, e $20 \%$ (2) dos estudos com uma perspectiva da saúde suplementar, organizações privadas (Quadro 2).

Verificou-se que 100\% (10) dos estudos apontaram dominância para a intervenção; que 40\% (4) não apresentaram claramente o horizonte de tempo da avaliação econômica. Pode-se constatar que 80\% (8) dos estudos apontaram a análise de custo efetividade como tipo de avaliação econômica, e $20 \%$ (2) dos estudos apontaram a análise de custo minimização como tipo de avaliação econômica. Observou-se que quanto ao tipo de intervenção $30 \%$ (3) avaliaram medicamentos, $20 \%$ (2) avaliaram correlatos, $20 \%$ (2) avaliaram testes de diagnósticos, $20 \%$ (2) avaliaram programas e custos de diagnósticos, e 10\% (1) avaliou terapia nutricional (Quadro 2).

\section{Discussão}

Os artigos apresentam informações e dados consistentes, relevantes, oferecem bons argumentos aos gestores e contribuem para as decisões de acordo com as perspectivas de seus objetivos; contudo se faz necessário observar e evoluir com a utilização dos conceitos, do escopo teórico, da economia da saúde para uma metodologia eficaz quanto aos objetivos de estudos de avaliação econômica na saúde.

Os vários modelos de avaliação econômica se aplicam a todos os tipos de tecnologias da saúde, incluindo drogas, materiais, procedimentos, sistemas e organizações para cuidados da saúde ${ }^{16}$. Quando são utilizados de forma estratégica os gestores são efetivos em suas decisões, otimizando a aplicação dos recursos de suas instituições, oferecendo uma qualidade da assistência com maior segurança e qualidade, e influenciando positivamente o acesso da população ao sistema de saúde.

Diante do Gráfico 1 observou-se uma linha de tendência linear apontando para uma diminuição na quantidade de estudos desenvolvidos sobre o tema, evento que desafia o sistema de saúde, suas organizações, seus gestores, diante da necessidade de buscar eficiência e efetividade na gestão dos recursos, já que para tomada de decisões assertivas as avaliações econômicas são ferramentas chaves do processo, sendo uma análise comparativa de ações alternativas em termos de seus custos e consequências, sendo seus componentes básico o identificar, quantificar, avaliar e comparar os custos e consequências das alternativas ${ }^{17}$.

O Gráfico 2 apresenta a distribuição dos artigos elegíveis por estado brasileiro, visualiza-se que além dos poucos estudos sobre o tema, também poucos estados se apresentam como bases de desenvolvimento das avaliações econômicas de tecnologia nos serviços de saúde, supõe-se, a partir dessas informações, fortes limitações no processo de decisão dos gestores de saúde. Segundo Drummond, Iglesias e Rovira ${ }^{18}$, artigo publicado em 2005, para acelerar o processo de incorporação das avaliações econômicas como uma ferramenta formal para embasar os processos de tomada de decisão dentro dos sistemas de saúde nos países da América Latina, duas condições principais precisam ser atendidas, primeiro, recursos e habilidades adequados precisam estar disponíveis para conduzir avaliações econômicas de boa qualidade; em segundo lugar, os procedimentos de tomada de decisão precisam ser modificados para acomodar abordagens "baseadas em evidências", como as avaliações econômicas.

Outro achado demonstra fragilidade no processo de tomada de decisão de gestão, baseado em evidências, das organizações de saúde privadas no Brasil, observou-se que 80\% (8) dos estudos encontrados apontaram suas perspectivas para o SUS (Sistema Único de Saúde do Brasil), enquanto que 20\% (2) apontaram suas perspectivas para saúde suplementar, cuja 
importância para a sustentabilidade do setor é alta, em 2020 o setor registrou mais de 70 milhões de beneficiários, e suas operadoras registraram receita de aproximadamente 160 bilhões de reais ${ }^{19}$.

Percebe-se nos achados que 40\% (4) dos estudos não apresentaram o horizonte de tempo necessário para encontrar os valores dos resultados das intervenções; isso implica numa dificuldade da valoração dos recursos e dos resultados, indicadores fundamentais para as conclusões das avaliações econômicas.

A avaliação econômica de uma tecnologia deve tomar como base o curso natural da condição e o provável impacto que a intervenção tem sobre ele. A decisão do horizonte temporal da análise deve ser capaz de capturar todas as consequências e os custos relevantes para a medida de resultado escolhida. Dessa forma, deve ser explicitado e justificado o horizonte temporal da avaliação. Para doenças crônicas e aquelas em que diferenças na mortalidade são significativas, os estudos devem considerar a expectativa de vida dos pacientes como horizonte temporal prioritário. Para doenças ou condições agudas, sem sequelas a longo prazo, o menor período que permita avaliar os desfechos relevantes é indicado (como exemplo, um horizonte temporal curto seria suficiente para avaliar resolução de estomatite herpética em crianças $)^{20}$.

Observou-se que $100 \%$ (10) dos estudos concluíram dominância para intervenção; $80 \%$ (8) utilizaram o tipo análise de custo efetividade e $20 \%$ (2) utilizaram o tipo análise de custo minimização, não se verificou estudos com análise de custo utilidade e análise de custo-benefício. Verificou-se algumas lacunas no desenvolvimento desses estudos, as principais foram a valoração dos custos com mão de obra, alguns deles não consideraram as análises de sensibilidade e os impactos nas vidas dos principais beneficiários das intervenções, importante essa perspectiva, pois segundo a OMS (Organização Mundial de Saúde) saúde é o estado de completo bem-estar físico, mental e social, e não apenas a ausência de doença.

Detecta-se nos achados uma escassez de avaliações econômicas para as intervenções com dispositivos de acesso vasculares, considerando que $90 \%$ dos pacientes que se internam nos serviços de saúde hospitalar recebem um dispositivo vascular, supõe-se que esse grupo de recursos representam uma parcela significativa nos orçamentos das organizações de saúde.

\section{Considerações Finais}

A contribuição desse estudo foi de pesquisar na literatura existente as principais avaliações econômicas de tecnologias utilizadas nos serviços de saúde do Brasil, apesar dos poucos estudos encontrados nas bases de pesquisa, todos apresentaram resultados impactantes e consistentes. Contudo essa revisão encontrou algumas lacunas do conhecimento; verificou-se como pontos potenciais de melhorias para as próximas avaliações, a metodologia, os tipos das avaliações econômicas, valoração dos recursos como mão de obra, análises de sensibilidade, resultados e indicadores econômicos em saúde, como qualidade de vida, curas, desospitalizações, hospitalizações, anos ajustados a qualidade de vida, e outros dados que podem representar valor para o paciente como o retorno antecipado a atividade econômica ou seu adiamento devido ao período de tratamento da enfermidade, assim como alguns custos não foram considerados em alguns estudos, como valoração de recursos humanos, tempo de internação e custos com performance dos dispositivos avaliados.

\section{Referências}

Arksey H \& O’Malley L (2005). Scoping studies: towards a methodological framework. Int J Soc Res Methodol. https://doi.org/10.1080/1364557032000119616

Armstrong R., Hall B.J., Doyle J. \& Waters E. (2011) Cochrane Update. 'Scoping the scope' of a cochrane review. Journal of public health. 33(1):147-150. https://doi.org/10.1093/pubmed/fdr015

Brasil. Ministério da Saúde. Resolução RDC nº 36, de 25 de julho de 2013. Institui ações para a segurança do paciente em serviços de saúde e dá outras providências. Diário Oficial da União, Brasília, DF, 2013. 
Brasil. Ministério da Saúde. Agência Nacional de Saúde Suplementar. Receitas e despesas. ANS; 2021. http://www.ans.gov.br/acesso-a-informacao/receitas-edespesas

Brasil. Ministério da Saúde. (2014). Diretrizes metodológicas: Diretriz de Avaliação Econômica. Secretaria de Ciência, Tecnologia e Insumos Estratégicos; https://bvsms.saude.gov.br/bvs/publicacoes/diretrizes_metodologicas_diretriz_avaliacao_economica.pdf

Correia M. I. T. D., Castro M., Toledo D. O., Farah D., Sansone D., Morais T. R. A. \& Araújo G. T. B., Fonseca M. (2020). Nutrition Therapy CostEffectiveness Model Indicating How Nutrition May Contribute to the Efficiency and Financial Sustainability of the Health Systems. JPEN. Journal of parenteral and enteral nutrition. 1(11):1-9. https://doi.org/10.1002/jpen.2052

Drummond M. F., Sculpher M. J., Claxton K., Stoddart G. L. \& Torrance G. W. (2015). Methods for the Economic Evaluation of Health Care Programmes. Oxford: Oxford University Press.

Drummond M. F., Sculpher M. J., Torrance G., O. 'Brien B. \& Stoddart, G. (2002). Methods for The Economic Evaluation of Health Care Programmes. Oxford: Oxford Medical Publications.

Gomes M. \& Romcy H. (2005). Avaliação econômica da utilização de seringa pré-enchida versus seringa preenchida manualmente para flushing em pacientes com cateter venoso central na perspectiva de operadoras de saúde. J bras econ saúde. 10(3):239-245. DOI: 10.21115/JBES.v10.n3.p239-45

Iglesias C. P., Drummond M. F., Rovira J. \& Nevalat Project Group (2005). Health-care decision-making processes in Latin America: problems and prospects for the use of economic evaluation. International journal of technology assessment in health care. 2005;21(1):1-14. https://doi.org/10.1017/s0266462305050014

Marcolino, M. S., Polanczyk, C. A., Bovendorp, A. C., Marques, N. S., Silva, L. A., Turquia, C. P. \& Ribeiro, A. L. (2016). Economic evaluation of the new oral anticoagulants for the prevention of thromboembolic events: a cost-minimization analysis. Sao Paulo medical journal = Revista paulista de medicina, 134(4), 322-329. https://doi.org/10.1590/1516-3180.2016.0019260216

Mattos Â. Z., Mattos A. A. \& Ribeiro R. A. (2016). Rerlipressin versus noradrenaline for hepatorenal syndrome: economic evaluation under the perspective of the Brazilian Public Health System. Arquivos de gastroenterologia. 53(2):123-126. https://doi.org/10.1590/S0004-28032016000200014

Meirelles I. O., Couto D. H. N. \& Costa R. S. (2019). Custo-efetividade do pazopanibe comparado ao sunitinibe para câncer renal metastático na perspectiva. Cad Saude Publica. 35(8):1-9. https://doi.org/10.1590/0102-311X00108218

Miranda, M. C., Haddad L., Trindade E., Cassenote A., Hayashi G. Y., Damiani D., Costa F. C., Madureira G., Mendonca B. B. \& Bachega T. (2021). The Cost-Effectiveness of Congenital Adrenal Hyperplasia Newborn Screening in Brazil: A Comparison Between Screened and Unscreened Cohorts. Frontiers in pediatrics. 9(1):1-13. https://doi.org/10.3389/fped.2021.659492

Oliveira R., Mourão T. C., Santana T., Favaretto R. L., Zequi S. C. \& Guimarães G. C. (2021). Cost-Effectiveness Analysis of Prostate Cancer Screening in Brazil. Value in health regional issues. 26(1):89-97. https://doi.org/10.1016/j.vhri.2021.02.002

Peixoto H. M., Brito M. A. M., Romero G. A. S., Monteiro W. M., Lacerda M. V. G. \& Oliveira M. R. F. (2016). Cost-effectiveness analysis of rapid diagnostic tests for G6PD deficiency in patients with Plasmodium vivax malaria in the Brazilian Amazon. Malar J. 15(2):1-13. https://doi.org/10.1186/s12936-016-1140-x

Peters M. D., Godfrey C. M., Khalil H., McInerney P., Parker D. \& Soares C. B. (2015). Guidance for conducting systematic scoping reviews. International journal of evidence-based healthcare. 13(3):141-146. https://doi.org/10.1097/XEB.0000000000000050

Tricco A. C., Lillie E., Zarin W., O. 'Brien K. K., Colquhoun H., Levac D., Moher D., Peters M. D., Horsley T., Weeks L. \& Hempel S. (2018) PRISMA Extension for Scoping Reviews (PRISMA-ScR): Checklist and Explanation. Ann Intern Med. 169(7):467-473. doi:10.7326/M18-0850.

Ubels J., Sable C., Beaton A. Z., Nunes M., Oliveira K., Rabelo L. C., Teixeira I. M., Ruiz G., Rabelo L., Tompsett A. R., Ribeiro A., Sahlen K. G. \& Nascimento B. R. (2020). PROVAR+ (Programa de RastreamentO da VAlvopatia Reumática e Outras Doenças Cardiovasculares) investigators. CostEffectiveness of Rheumatic Heart Disease Echocardiographic Screening in Brazil: Data from the PROVAR+ Study: Cost-effectiveness of RHD screening in Brazil. Global heart. 15(1):1-18. https://doi.org/10.5334/gh.529

Veras B. M., Senna K. M., Correia M. G. \& Santos M. S. (2013). Cost-effectiveness analysis on the reutilization of coronary artery catheters in a public hospital in Rio de Janeiro, Brazil. Cad Saude Publica. 29(1):110-120. doi: 10.1590/0102-311x00021813 